\title{
Intraoperative Scrub Nurse Handoffs Are Associated with Increased Operative Times for Lower Extremity Orthopaedic Sports Procedures
}

\author{
Bradley T. Hammoor, M.S., Austin C. Kaidi, M.Sc., Connor R. Crutchfield, B.A., \\ Xavier E. Ferrer, B.S., Thomas R. Hickernell, M.D., Christopher S. Ahmad, M.D., \\ William N. Levine, M.D., and T. Sean Lynch, M.D.
}

\begin{abstract}
Purpose: The purpose of this study was to evaluate the effect of intraoperative scrub nurse handoffs on surgical times for arthroscopically-assisted anterior cruciate ligament (ACL) reconstructions and hip arthroscopies. Methods: A retrospective chart review was done at a major, urban academic medical center for all patients who underwent arthroscopically-assisted ACL reconstructions and hip arthroscopies for femoroacetabular impingement syndrome between May 2014 and May 2020. All ACL reconstructions were performed by 1 of 6 sports medicine fellowship-trained surgeons, and all hip arthroscopies were performed by a single surgeon. Operative times, number of scrub nurse handoffs, surgeon, patient demographics, and procedure-specific information were recorded. The association between patient characteristics and the number of handoffs, as well as the association between patient characteristics and operative times, stratified by scrub nurse handoffs, were calculated. A multivariable linear regression was performed to assess the association between intra-operative handoffs and operative times. Results: Eight hundred twenty ACL reconstructions and 269 hip arthroscopies were identified. Multivariable linear regression demonstrated increasing intraoperative scrub nurse handoffs were associated with increased operative times for all patients. For ACL reconstructions, when including all possible covariates, 1 scrub nurse handoff increased operative times by 21.1 minutes (95\% confidence interval [CI]: 15.36 to $26.89 ; P<.001)$, and $2+$ handoffs increased operative times by 34.2 minutes (95\% CI: 26.28 to $42.15 ; P<.001)$. For hip arthroscopies, 1 scrub nurse handoff increased operative times by 7.0 minutes $(95 \%$ CI: 0.31 to $13.74 ; P=.04)$. Conclusion: Although a causal link cannot be made, intraoperative scrub nurse handoffs were associated with statistically significant increase in operative times for both ACL reconstructions and hip arthroscopies. Level of Evidence: Level III, retrospective cohort study.
\end{abstract}

A successful and efficient surgical operation is dependent on the collaboration of multiple interdisciplinary healthcare professionals. This is especially

From the Department of Orthopedic Surgery, Columbia University Medical Center, New York, New York, U.S.A.

B.T.M. and A.C.K. are co-first authors.

The authors report the following potential conflicts of interest or sources of funding: C.S.A. reports grants from Arthrex Inc and Major League Baseball; personal fees from Arthrex Inc; and other from Arthrex Inc and At Peak. T.S.L. reports other from Smith $\theta$ Nephew and CONMED Linvatec. Full ICMJE author disclosure forms are available for this article online, as supplementary material.

Received December 17, 2020; accepted March 30, 2021.

Address correspondence to T. Sean Lynch, M.D., Department of Orthopedic Surgery, Columbia University Medical Center, 622 W. $168^{\text {th }}$ St, PH-11, New York, NY10032,U.S.A.E-mail:ts12120@cumc.columbia.edu

(C) 2021 THE AUTHORS. Published by Elsevier Inc. on behalf of the Arthroscopy Association of North America. This is an open access article under the CC BY-NC-ND license (http://creativecommons.org/licenses/by-nc-nd/4.0/). 2666-061X/201981

https://doi.org/10.1016/j.asmr.2021.03.016 true in orthopaedic operations, where complex, multistep surgical tools (like flip-cutters) are often used. In an orthopaedic operating room (OR), an attending orthopaedic surgeon, anesthesiologist, resident physician, physician's assistant, medical student, and circulating/ scrub nurses are often working together to provide patients with high-quality care. Although each of these providers plays a critical role, the staffing models and workflows can vary considerably.

Surgeons generally remain scrubbed in for an entire surgical procedure, whereas the assisting surgical nurses ("scrub nurses" or "scrub technicians") work in shifts. As a result, there is a need for intraoperative handoffs at breaks or shift-change. These exchanges can occur as frequently as every two hours at our large, academic institution and are commonplace in hospitals throughout the country, and can be potentially hazardous. The incoming scrub nurse may be unsure of where in the case the surgeon is and unprepared for the 
upcoming procedural steps. Few existing works in nonorthopaedic surgery literature have found that intra-operative scrub nurse handoffs can increase operative times. $^{1-4}$

The impact of other surgical staff interventions on operative efficiency has been well established, however, and existing literature has shown that OR staffing interventions are prime targets for improving patient outcomes. $^{5-8}$ In the orthopaedic literature for example, Murgai et al. ${ }^{9}$ showed that having dedicated orthopaedic scrub nurses decreases operative times and blood loss in adolescent idiopathic scoliosis surgery. ${ }^{9}$

For this reason, identifying structural factors that increase surgery length, like intraoperative handoffs, preoperative delays, postanesthesia care unit bed unavailability, and more can have a substantial impact on overall patient care by decreasing costs and surgical complication rates. ${ }^{10-12}$ After all, each minute in an OR costs $\$ 62$ on average and can be as high as $\$ 133$ per hour in states like New York, ${ }^{11}$ and 15 extra minutes can increase infection risk by $13 \% .^{12}$

In this study, we analyze the effect of intraoperative scrub nurse handoffs on two high-volume orthopaedic procedures, anterior cruciate ligament (ACL) reconstructions and hip arthroscopies. Arthroscopicallyassisted ACL reconstructions are among the most common orthopaedic sports medicine procedures; meanwhile, hip arthroscopy is the fastest growing arthroscopic procedure in the United States. ${ }^{13-15}$ This high volume makes these procedures prime targets for interventions that can decrease OR time and cumulatively decrease overall U.S. healthcare burdens. The purpose of this study was to evaluate the effect of intraoperative scrub nurse handoffs on surgical times for arthroscopically-assisted anterior cruciate ligament reconstructions and hip arthroscopies. We hypothesize that increased scrub nurse handoffs will be associated with significantly increased operative times of both of these complex procedures.

\section{Methods}

\section{Cohort Selection}

This study was designed as a retrospective cohort study. The study population was identified via chart review from our large $(700+$ bed), urban, academic medical center. All patients who underwent arthroscopically-assisted ACL reconstruction or hip arthroscopy between May 2014 and May 2020 were identified via searching billing data for Current Procedural Terminology (CPT) codes 29888 and 29914, 29915, and 29116. These codes corresponded to ACL reconstructions, hip arthroscopy with femoroplasty, hip arthroscopy with acetabuloplasty, and hip arthroscopy with labral repair, respectively. All operations were done as outpatient procedures at the hospital. All ACL reconstructions were done by 6 sports medicine fellowship-trained surgeons. All hip arthroscopies were done by a single surgeon (T.S.L.) who specializes in hip arthroscopy procedures.

For all cases, patients were excluded if there were greater than four intra-operative scrub nurse handoffs and if they had American Society of Anesthesiologists class (ASA) scores of 3 to 4 , because these likely represented complex cases that could confound any handoff effects. To ensure comparable surgeon competency, patients were included only if their surgeon completed an orthopedic sports medicine fellowship. Operative times, number of scrub nurse handoffs, operating surgeon's fellowship status, and patient demographical information (age, sex, body mass index, ASA score, self-reported race, language and insurance status) were recorded for each patient.

For ACL reconstruction patients, the following additional information was collected: primary versus revision, graft type (allograft, autograft, or a hybrid), whether a concomitant meniscal repair was done on the medial, lateral, or both menisci, whether a concomitant partial meniscectomy was done on the medial, lateral, or both menisci, and whether any other concomitant ligament procedures were performed. For hip arthroscopy patients, procedural components including whether each patient received a labral repair, acetabuloplasty, and/or femoroplasty were recorded.

To ensure our study was appropriately powered, a priori and post-hoc power analyses were conducted using an estimated effect size from the literature of $10 \%{ }^{2}$-both demonstrated greater than $95 \%$ power with our expected sample size. Before all analyses, data normality was grossly assessed using histography. This study's protocol was approved by our institutional review board.

\section{Scrub Nurse Staffing and Training}

At our institution, scrub nurses handoff approximately every 2 hours for mandated breaks, and no restrictions exist on when handoffs may occur-that is, during ACL tunnel drilling, hip labral repair, and more. Our orthopaedic scrub nurses are trained in assisting arthroscopic procedures and are usually the primary scrub nurse for these surgeries. Occasionally, a nonorthopaedic scrub nurse will relieve the primary scrub nurse for these breaks. Scrub nurses at our institution require a nursing degree or scrub technician certificate, but procedure-specific training is informal and done by shadowing more senior scrub nurses who specialize in orthopaedic procedures. The scrub nurse specialty is not recorded in any hospital documentation.

\section{Association between Baseline Characteristics, Exposure, and Outcome}

First, we analyzed the association between patient demographic/procedure-specific variables with our 
Table 1. ACL Reconstruction Patient Demographics $(\mathrm{n}=820)$

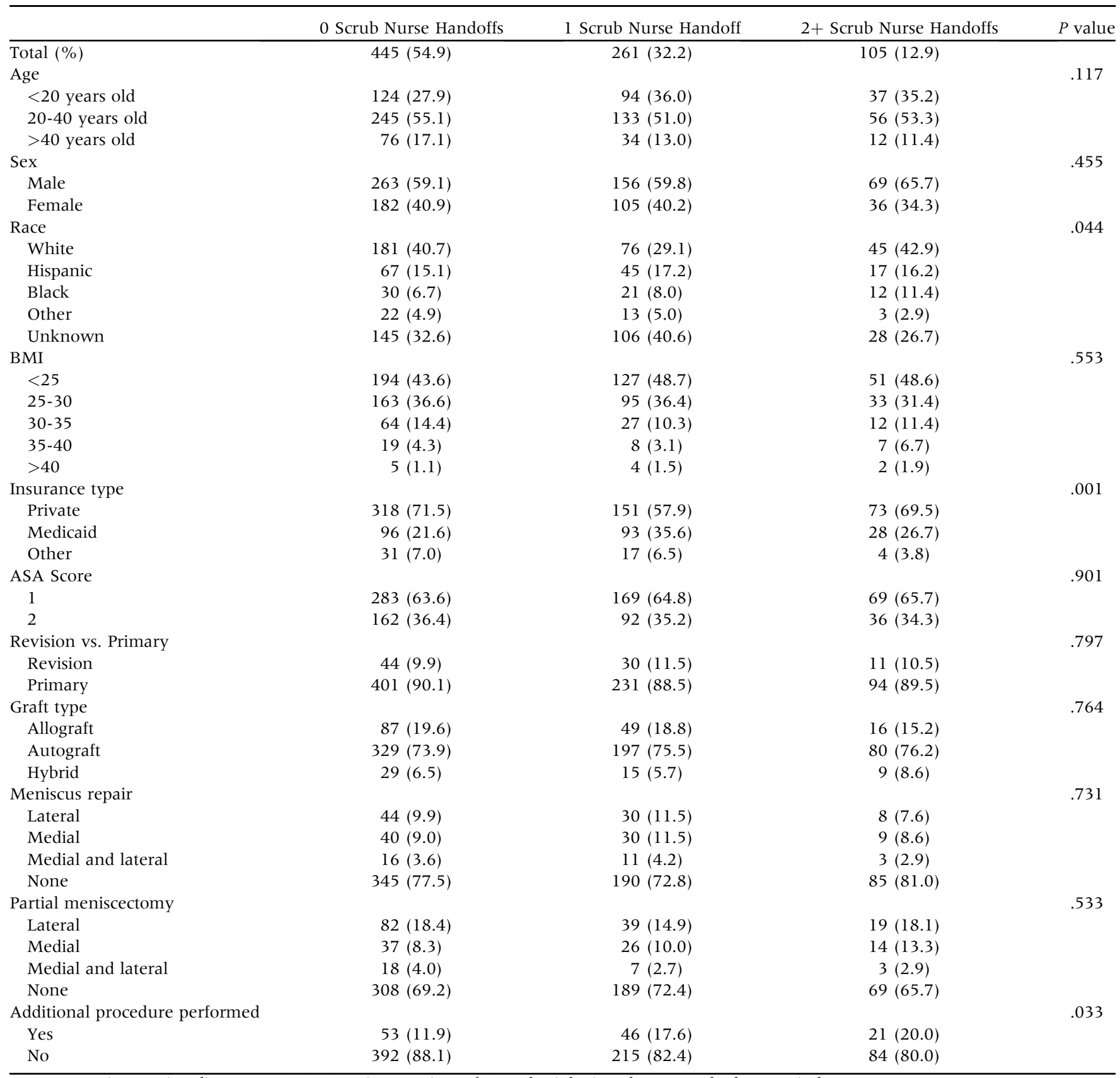

ACL, anterior cruciate ligament; ASA, American Society of Anesthesiologists class; BMI, body mess index.

exposure (scrub nurse handoffs) and outcome (operative times) independently. The number of scrub nurse handoffs was treated as a categorical variable and divided into cases with 0 intraoperative scrub nurse handoffs, 1 intraoperative scrub nurse handoffs, or $2+$ intraoperative handoffs. The associations between baseline, demographic characteristics and scrub nurse handoffs were analyzed using $\chi^{2}$ tests in $\mathrm{R}$ (version 3.6.2; Rstudio Inc, Boston, MA). Operative time was treated as a continuous variable. The associations between baseline characteristics were stratified by number of scrub nurse handoffs and operative time was analyzed using 1-way analyses of variance in $\mathrm{R}$ (version 3.6.2, Rstudio Inc). $P$ values $<.05$ were considered significant.

\section{Multivariable Linear Regression}

A multivariable linear regression (MLR) model was then built to determine the effect of each variable on operative times as well as control for possible confounders identified in prior analysis. Coefficients with 95\% confidence intervals were calculated for each variable to estimate effect size on operative times. 
Table 2. Hip Arthroscopy Patient Demographics $(\mathrm{n}=269)$

\begin{tabular}{|c|c|c|c|}
\hline & $\begin{array}{c}0 \text { Scrub } \\
\text { Nurse } \\
\text { Handoffs }\end{array}$ & $\begin{array}{l}1 \text { Scrub } \\
\text { Nurse } \\
\text { Handoff }\end{array}$ & $P$ value \\
\hline Total, no. (\%) & $181(67.3)$ & $88(32.7)$ & \\
\hline Age, no. (\%) & & & .214 \\
\hline$<20$ years old & $38(21.0)$ & $14(15.9)$ & \\
\hline 20-40 years old & $123(68.0)$ & $58(65.9)$ & \\
\hline$>40$ years old & $20(11.0)$ & $16(18.2)$ & \\
\hline Sex, no. (\%) & & & .345 \\
\hline Male & $82(45.3)$ & $46(52.3)$ & \\
\hline Female & $99(54.7)$ & $42(47.7)$ & \\
\hline Race, no. (\%) & & & .230 \\
\hline White & $97(53.6)$ & $52(59.1)$ & \\
\hline Hispanic & $14(7.7)$ & $5(5.7)$ & \\
\hline Black & $8(4.4)$ & $5(5.7)$ & \\
\hline Other & $3(1.7)$ & $5(5.7)$ & \\
\hline Unknown & $59(32.6)$ & $21(23.9)$ & \\
\hline BMI, no. (\%) & & & .096 \\
\hline$<25$ & $121(66.9)$ & 47 (53.4) & \\
\hline $25-30$ & $42(23.2)$ & $30(34.1)$ & \\
\hline $30-35$ & $18(9.9)$ & $11(12.5)$ & \\
\hline $35-40$ & $172(95.0)$ & $74(84.1)$ & \\
\hline$>40$ & $4(2.2)$ & $6(6.8)$ & \\
\hline Insurance type, no. (\%) & & & .011 \\
\hline Private & $172(95.0)$ & $74(84.1)$ & \\
\hline Medicaid & $4(2.2)$ & $6(6.8)$ & \\
\hline Other & $5(2.8)$ & $8(9.1)$ & \\
\hline ASA Score, no. (\%) & & & .684 \\
\hline 1 & $105(58.0)$ & $48(54.5)$ & \\
\hline 2 & $76(42.0)$ & $40(45.5)$ & \\
\hline Labral repair performed? & & & .088 \\
\hline Yes & $175(96.7)$ & $80(90.9)$ & \\
\hline No & $6(3.3)$ & $8(9.1)$ & \\
\hline Acetabuloplasty performed? & & & .406 \\
\hline Yes & $179(9 s 8.9)$ & $85(96.6)$ & \\
\hline No & $2(1.1)$ & $3(3.4)$ & \\
\hline Femoroplasty performed? & & & .636 \\
\hline Yes & $178(98.3)$ & $85(96.6)$ & \\
\hline No & $3(1.7)$ & $3(3.4)$ & \\
\hline
\end{tabular}

ASA, American Society of Anesthesiologists class; BMI, body mass index.

\section{Results}

Our search identified 820 ACL reconstruction patients and 269 hip arthroscopy patients (Tables 1 and 2). Among the ACL reconstruction patients, 445 $(54.9 \%)$ had 0 intra-operative scrub nurse handoffs, $261(32.2 \%)$ had 1 handoff, and 105 (12.9\%) had 2+ handoffs. Mean operative times among each group were 93.8 minutes ( \pm 38.6 standard deviation [SD]), 122.0 minutes $( \pm 44.4 \mathrm{SD})$, and 134.0 minutes $( \pm 54.8$ SD) for 0,1 , and $2+$ handoffs, respectively. Increased scrub nurse handoffs were also statistically associated with higher concomitant "additional procedures" being performed (Table 1). For hip arthroscopy patients, 181 $(67.3 \%)$ had 0 intraoperative scrub nurse handoffs, and $88(32.7 \%)$ had 1 handoff. Very few hip arthroscopy cases had $2+$ handoffs, and these were excluded as the sample size was not large enough to be significant. Mean operative times for hip arthroscopy cases were 96.1 minutes $( \pm 23.2 \mathrm{SD})$ and 103.0 minutes $( \pm 28.3 \mathrm{SD})$ for those with 0 and 1 scrub nurse handoff, respectively.

Our MLR identified multiple variables that had a statistically significant impact on operative times. For ACL reconstructions, including all measured covariates, 1 scrub nurse handoff was associated with a 21.1-minute increase in operative times $(P<.001)$ and $2+$ scrub nurse handoffs were associated with a 34.2-minute increase in operative times $(P<.001)$. For hip arthroscopy patients, I handoff was associated with a 7.0-minute increase in operative times $(P=.04)$. Subgroup analysis showed these increased operative times were significant across multiple groups (Figs 1 and 2).

\section{Discussion}

In this study, we found that scrub nurse handoffs were associated with increased operative times for ACL reconstructions and hip arthroscopies. This is consistent with findings in the limited existing literature. Giugale et al. studied the effect of circulating and scrub nurse handoffs on sacrocolpopexy operative times. They found each scrub nurse handoff was associated with a 13.6-minute increase in OR time. ${ }^{2}$ Additionally, among gynecologic oncology patients, Doll et al. found that one scrub nurse handoff increased the odds of a surgical complication by $\times 2.12$. $^{1}$ Among cardiac surgery procedures, Bloom et al. ${ }^{4}$ found that $2+$ scrub nurse handoffs were associated with a significantly increased rate of sharps-count errors. This is important because the authors showed an incorrect sharps-counts caused an average 1.3 hours delay in OR time and increased a patient's inhospital mortality odds ratio. Interestingly enough, of the 732 cases with an incorrect sharps count, none of these instances yielded radiographic evidence of any retained in the patient's body. This study analyzed the effect of these abundant handoffs on operative times for orthopaedic procedures.

In this level III, retrospective study, our findings supported the hypothesis that intraoperative scrub nurse handoffs increase operative times for ACL reconstructions and hip arthroscopies. Overall, intraoperative handoffs were associated with significantly increased surgical times across many subgroups for both ACL reconstruction and hip arthroscopy patients. For many sub-groups, these time differences were significant when comparing the 0 and 1 intra-operative handoff groups, the 1 and $2+$ handoff group, and the overall multivariable analyses of variance (Figs 1 and 2).

To control for the patient and procedure specific factors that can influence operative times, this study first identified confounding variables. For ACL reconstruction patients, race, insurance type, and performing 
Fig 1. Operative times (minutes) for anterior cruciate ligament reconstruction patient demographic groups, stratified by number of intraoperative scrub nurse handoffs $(0,1,2+)$. Findings indicate that scrub nurse handoffs were significantly associated with increased operative times for multiple demographic groups. Error bars represent $95 \%$ confidence intervals. $P$ values for 1 -way analysis of variance for each group is listed on right of grouped columns.

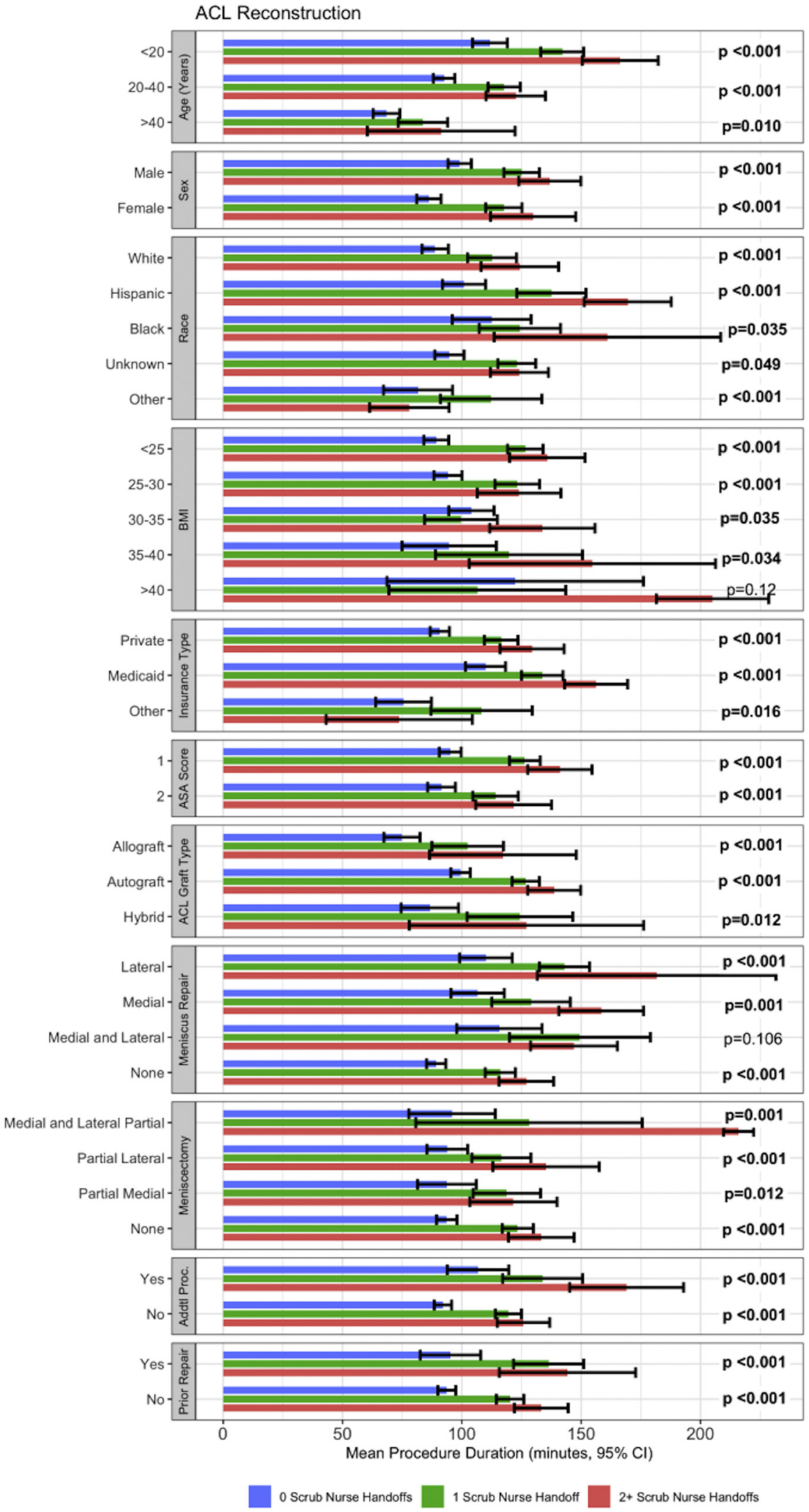




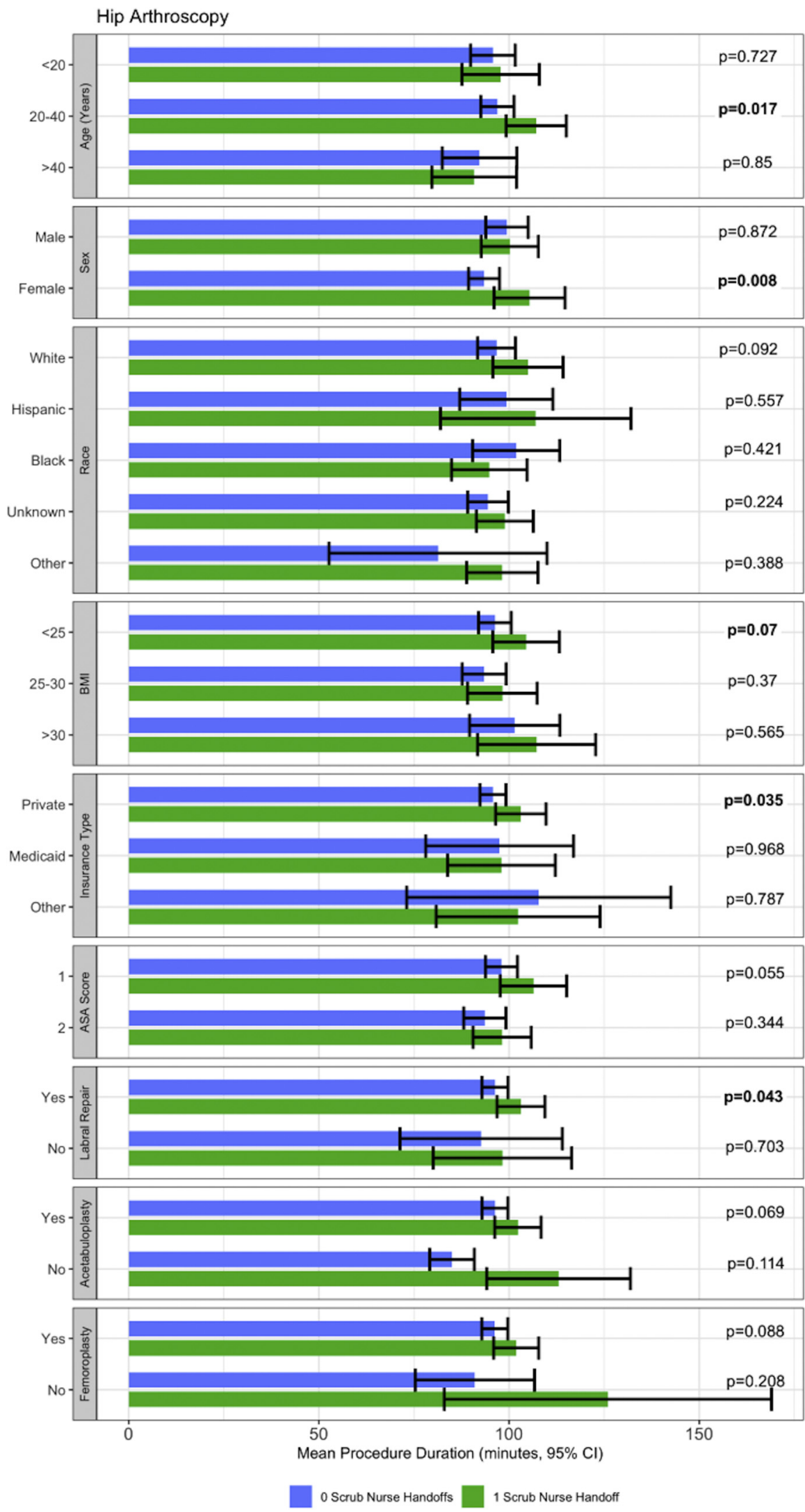

Fig 2. Operative times (minutes) for hip arthroscopy patient demographic groups, stratified by number of intraoperative scrub nurse handoffs (0 and 1$)$. Findings indicate that scrub nurse handoffs were significantly associated with increased operative times for multiple demographic groups. Error bars represent 95\% confidence intervals. $P$ values for 1 -way analysis of variance for each group is listed on right of grouped columns.

Scrub Nurse Handoffs

Scrub Nurse Handof 
another procedure were confounding variables. No confounding variables were identified for hip arthroscopy patients. After controlling for confounding variables, 1 and 2+ intraoperative scrub nurse handoffs were associated with 21.1-minute and 34.2-minute increases in operative times among ACL reconstruction patients. Among the hip arthroscopy group, 1 intraoperative scrub nurse handoff increased operative times by 7.0 minutes.

These remarkable increases in operative times may be because ACL reconstructions and hip arthroscopies require a precise orchestration of equipment setup and procedural anticipation to avoid surgical delays. This can only be accomplished seamlessly when an experienced and engaged scrub tech is present. These procedures' reliance on an integral, educated, and motivated assistant make it easy for staff-related increases in operative times to occur. For example, if a scrub nurse knows they will be relieved 15 minutes into a case, they may not spend as much time properly setting up the back table. Additionally, inconsistent handoff sign-outs could result in a scrub tech who must take additional time to identify what procedural steps have already taken place. Each of these small missteps can cumulatively create a significant impact on operative times. Although the primary outcome studied in this article is operative time, the downstream effects of these intraoperative handoffs should not be understated. Increased time in an OR increases the amount billed to a patient and their risk of a surgical site infection. ${ }^{1,12}$

\section{Limitations}

Although many steps were taken to account for confounding variables and study bias, this study still has multiple inherent limitations. First, the retrospective nature of this study makes it impossible to determine a causal link between intraoperative handoffs and increased operative times. Although the small increase in operative times caused by a single scrub nurse handoff is unlikely to overlap with a shift-change, no definitive causation can be stated, and hospital staffing policies make randomized studies impossible to carry out. Second, one variable that was unable to be accounted for was scrub nurse specialty. Although the majority of scrub nurses who assist with orthopaedic procedures at our institution are dedicated orthopaedics scrub nurses, occasionally scrub nurses from other surgical specialties will relieve the primary scrub nurse. Scrub nurses unaccustomed to the complicated equipment used for arthroscopy could have a significant effect on operative times. Third, this study did not evaluate the effect of scrub nurse handoffs on patient outcomes. Fourth, although a significant number of potential case-specific variables were built into our MLR, unaccounted for operation-specific factors (like surgeon annual case volume and case complexity) could influence our results.

\section{Conclusions}

Although a causal link cannot be made, intraoperative scrub nurse handoffs were associated with statistically significant increases in operative times for both ACL reconstructions and hip arthroscopies.

\section{References}

1. Doll KM, Lavery JA, Snavely AC, Gehrig PA. Intraoperative handoffs and postoperative complications among patients undergoing gynecologic oncology operations. J Healthe Qual 2017;39:e42-e48.

2. Giugale LE, Sears S, Lavelle ES, Carter-Brooks CM, Bonidie M, Shepherd JP. Evaluating the impact of intraoperative surgical team handoffs on patient outcomes. Female Pelvic Med Reconstr Surg 2017;23:288-292.

3. Talsma AN, Anderson C, Geun HG, Guo Y, Campbell DA. Evaluation of OR staffing and postoperative patient outcomes. AORN J 2013;97:230-242.

4. Bloom JP, Moonsamy P, Gartland RM, et al. Impact of staff turnover during cardiac surgical procedures [published online November 30, 2019]. J Thorac Cardiovasc Surg. https://10.1016/j.jtcvs.2019.11.051

5. Epstein RH, Dexter F, Gratch DM, Lubarsky DA. Intraoperative handoffs among anesthesia providers increase the incidence of documentation errors for controlled drugs. Jt Comm J Qual Patient Saf 2017;43:396-402.

6. Jones PM, Cherry RA, Allen BN, et al. Association between handover of anesthesia care and adverse postoperative outcomes among patients undergoing major surgery. JAMA 2018;319:143-153.

7. Hudson CCC, McDonald B, Hudson JKC, Tran D, Boodhwani M. Impact of anesthetic handover on mortality and morbidity in cardiac surgery: A cohort study. J Cardiothorac Vasc Anesth 2015;29:11-16.

8. Sewell M, Adebibe M, Jayakumar P, et al. Use of the WHO surgical safety checklist in trauma and orthopaedic patients. Int Orthop 2011 ;35:897-901.

9. Murgai RR, Andras LM, Nielsen E, Scott G, Gould H, Skaggs DL. Dedicated spine nurses and scrub technicians improve intraoperative efficiency of surgery for adolescent idiopathic scoliosis. Spine Deform 2020;8: 171-176.

10. Dhupar R, Evankovich J, Klune JR, Vargas LG, Hughes SJ. Delayed operating room availability significantly impacts the total hospital costs of an urgent surgical procedure. Surgery $2011 ; 150: 299-305$.

11. Macario A. What does one minute of operating room time cost? J Clin Anesth 2010;22:233-236.

12. Cheng H, Chen BPH, Soleas IM, Ferko NC, Cameron CG, Hinoul P. Prolonged operative duration increases risk of surgical site infections: A systematic review. Surg Infect (Larchmt) 2017;18:722-735.

13. Buller LT, Best MJ, Baraga MG, Kaplan LD. Trends in anterior cruciate ligament reconstruction in the United States. Orthop J Sport Med 2015;3:1-8. 
14. Bozic KJ, Chan V, Valone FH, Feeley BT, Vail TP. Trends in hip arthroscopy utilization in the United States. J Arthroplasty 2013;28:140-143 (8 Suppl).
15. Zhang AL, Feeley BT. Editorial commentary: The rise of hip arthroscopy: Temporary trend or here to stay? Arthroscopy 2018;34:1831-1832. 